\title{
LA BOTÁNICA EN EL JARDÍN DE MIGAS CALIENTES
}

\author{
Susana Pinar \\ Miguel Angel Puig-Samper
}

Dpto. de H.a de la Ciencia - Centro de Estudios Históricos, CSIC - C/ Duque de Medinaceli 6, Madrid

\section{RESUMEN}

En este artículo se aportan nuevos datos sobre la constitución física del antiguo Jardín Botánico de Migas Calientes, fundado en 1755, de acuerdo a la documentación inédita conservada en la Biblioteca del Palacio de Santa Cruz de Valladolid. Hemos elaborado un nuevo análisis de la labor educativa de sus profesores y publicamos la relación de la biblioteca botánica de José Quer, situada en el actual jardín Botánico de Madrid, añadiendo, en todos los casos posibles, la identificación de las obras que se mencionan.

\section{SUMMARY}

In this article new information on the physical constitution of the old Botanical Garden of Calientes Migas Calientes, established in 1755, are contributed, according to the unpublished documentation conserved in the Library of the Palace of Santa Cruz de Valladolid. We have elaborated a new analysis of the educational work of its professors and we published the relationship of the botanical library of José Quer, located in the actual Botanical Garden of Madrid, adding, in all the possible cases, the identification of the works which are mentioned.

Las Memorias de la Academia Médica Matritense nos muestran que fue en el seno de esta institución, creada en 1734 , donde se fraguaron «los ventajosos designios de los Establecimientos del Jardín Botánico y Laboratorio Chimico, y el plantel de todos sus catedráticos...»' ${ }^{1}$. Concretamente en relación con la fundación del Real Jardín Botánico de Migas Calientes, destaca la intervención de José Ortega, Boticario Mayor de los Reales Ejércitos y Secretario Perpetuo del citado centro, quien hizo patente a su Presidente, José Suñol, la necesidad de establecer un jardín botánico que cubriera los requerimientos de la ciencia médica situándola a nivel europeo.

1 Memorias de la Real Academia Médica de Madrid, Imprenta Real, Madrid, 1797, tomo $1^{\circ}$, pp.ILII, cita en pág. XIV; también: PUIG SAMPER, M.A. (1987), «La enseñanza de la botánica en la España Ilustrada: El Jardín Botánico de Madrid» en SÁNCHEZ, A., Puig SAMPER, M.A. \& J. DE LA SOTA (eds.), La Real Expedición Botánica a Nueva España. Quinto Centenario, Madrid, pp.59-78. 
De este modo, el 17 de octubre de 1755, Ricardo Well firmaba la Real Resolución de Fernando VI para la erección del nuevo jardín, dejando la intendencia en manos de José Suñol y la subdirección a José Ortega y José Toledano, este último Boticario Real y miembro también de la Academia Médica. El puesto de primer profesor lo cubriría José Quer y el de segundo Juan Minuart, Cirujano Consultor y Boticario del Ejército respectivamente, ambos miembros académicos ${ }^{2}$.

\section{CONSTITUCIÓN FÍSICA DEL JARDÍN DE MIGAS CALIENTES}

Creado para la enseñanza de la botánica tanto en su vertiente teórica como en la práctica, el Jardín Botánico de Migas Calientes fue fundado en 1755 en la Huerta del mismo nombre, situada frente al Soto de Migas Calientes, a la derecha del camino de El Pardo. Aunque su emplazamiento se encuentra más o menos precisado, lo cierto es que el jardín existía ya desde el siglo XVI como heredad de un noble personaje, pasando a manos del boticario de cámara Luis Riqueur en mayo de $1713^{3}$, bajo las funciones de huerto frutal y cultivo de plantas medicinales, quien lo cedió al rey el 8 de junio de 1724, época a la que pertenecen los dos planos conocidos del jardín ${ }^{4}$.

Para su establecimiento, en 1747 se ordenó arrancar todas las plantas medicinales y trasladarlas al jardín de la Priora, dando así comienzo a las obras sobre el antiguo trazado. Se construyó un nuevo invernáculo en 1766; se arrancaron más de 800 árboles, formándose 12 cuadros grandes y cuatro parterres; se cercó de cañas y estacas de pino todo el jardín y se cerraron los parterres con llave. Hileras de boj se plantaron a las márgenes de las calles y se sustituyeron muchas de las plantas precedentes por aquéllas que Quer llevó de su jardín y las que se encontraban cerca de los Aflijidos 5 .

La siembra del jardín, en la que intervino Quer con las plantas traídas de sus excursiones por la Península, se dio por terminada en 1778. En el Índice impreso en 1772 aparecen unas 650 especies, más de la mitad españolas, cuyo número fue en

\footnotetext{
2 Sobre la fundación y constitución del Jardín véase igualmente: Colmeiro, M. (1875) Bosquejo histórico y estadístico del Jardín Botánico de Madrid. Imprenta de T. Fortanet, Madrid; Añón, C. (1987), Real Jardín Botánico de Madrid. Sus orígenes: 1755-1781, Real Jardín Botánico, CSIC-Quinto Centenario, Madrid, pp.11-36.

3 Sobre los traspasos de propiedad del citado jardín véase Añón, C. (1987), p.21.

4 Plano del estado de la huerta cedida, antiguo Jardín Botánico de Migas Calientes, E. MARCHAND? (1724) y Plano del proyecto de mejora para el antiguo Jardín Botánico de Migas Calientes, E. Marchand (1724), ambos reproducidos en AÑóN, C. (1987), pp. 22 y 24; figs. 15 y 16, respectivamente.

5 QUER, J. (1762) Flora Española ó Historia de las plantas que se crian en España, Joachin Ibarra, Madrid, tomo I, p.60; también, AÑón, C. (1987), p.27.
} 


\section{LA BOTÁNICA EN EL JARDÍN DE MIGAS CALIENTES}

aumento conforme a los catálogos manuscritos de 1775, 1776, 1777 y 1778, oscilando siempre entre las 1.200 y 1.500 especies $^{6}$.

La situación del Jardín Botánico de Migas Calientes podría haber sido la siguiente. Visto desde Madrid quedaría emplazado a la derecha del camino de El Pardo, teniendo clara la localización del Soto y Prado de Migas Calientes a su izquierda, colindantes con la Huerta del Marqués de Guerra, futuro Palacio de la Moncloa. Zona que probablemente corresponda a la que en el Dibujo del camino desde el puente de Segovia hasta la primera encina del Monte de El Pardo, de Francisco Pérez Cano $(1743)^{7}$, aparece como «Jardín del Boticario», frente a la esquina del Soto y con salida directa al río según el mismo plano.

Conforme a las medidas del jardín que figuran en el título de propiedad ${ }^{8}$, las observaciones sobre la toma de aguas del arroyo por la parte alta del mismo y el hecho de existir una cascada, obviamente, situada en dirección aguas abajo, emplazan al jardín de la forma siguiente: «la fachada principal del mencionado Jardín que está al Camino del Real Sitio de El Pardo mirando al Soto de Migas Calientes, tenía doscientos ochenta y dos pies, a cuya distancia formanba un ángulo saliente y seguía la línea con doscientos once pies» que corresponde en el plano al lado más estrecho, opuesto a la casa, con una puerta en la esquina izquierda; «por el costado de mano derecha a mediodía seguía toda la línea recta con ochocientos pies», lado mayor que deja la casa del jardinero en el ángulo superior derecho; «por la siniestra que miraba al Norte cuatro cientos cuarenta y tres pies, a cuya distancia formaba un ángulo entrante y seguía la línea con trescientos seis pies», uno de los lados más largos que deja el cenador y estanque en la esquina superior izquierda y la salida de aguas en la esquina inferior del mismo lado; «y por el testero que miraba a Oriente, toda su linea recta quinientos sesenta pies, con lo que quedaba cerrado el sitio y dentro de él se hallaba una casa con sólo una crujía y alzado de cuarto principal, y a la derecha de ésta otra de cuartos pequeños de teja vana, un Ivernáculo en el ángulo del testero, a la izquierda un cenador con cubiertas a cuatro aguas con dos entradas y Arcos que arrancaban de una columna de piedra berroqueña con asientos de la misma...»; todos los objetos ornamentales aparecen en la posición que se describen, casas e invernáculo en la esquina superior derecha, mientras en la esquina opuesta estaría el cenador.

- Colmeiro, M. (1875), pp.6-7; véanse también los catálogos de siembra que se conservan en el Real Jardín Botánico de Madrid.

7 Reproducido en AÑóN, C. (1987), pp.12-13, fig.4.

8 AÑ́N, C. (1987), pp.94-106, reproduce el documento conservado en el Archivo General de Palacio (A.G.P.), Títulos, leg. 1257/13, (1780, diciembre, 22). Escritura de la venta del Jardín Botánico, sito a las márgenes del río Manzanares, a favor de la excelentísima señora doña María Felipa Campbel, marquesa de Castejón, con una descripción detallada del mismo. 
Teniendo presente la remodelación del jardín sobre la planta de 1724, veámos ahora a grandes rasgos la constitución florística de los distintos parterres y cuadros del jardín y de los elementos decorativos que permanecieron tras su fundación, gracias a la información inédita conservada en la Biblioteca del Palacio de Santa Cruz de Valladolid (S.C.M.) ${ }^{9}$.

A la derecha de la calle principal que aparece en los documentos como «calle de enmedio» o «de los álamos», subiendo desde el río se encontraban los doce cuadros principales de las clases de Tournefort, compartiendo, por lo general, un par de clases por cuadro.

En medio de los cuadros se hallaba un estanquillo rodeado de plantas de muy diverso tipo entre las que destacan el drago, la Bignonia y la Indigofera tinctoria. En el ladó oeste, cercano al río, tiestos de diversos tamaños y continentes entre los que se cuentan varios aloes, opuntias, yucas, Cereus, etc. Al resguardo de la hoya formada con la tapia del jardín: Bryonia africana, Potentilla, Anagyris, Lysimachia, Aconitum, etc. En el lado izquierdo o norte del jardín se hallaban: un cuadro de patrones para injertar, otro de Hedera terrestris, dos de Viola, un bancal de Corona imperialis, 3 de Hyacinthus, cuatro cuadros de repuesto con bulbosas y anuales. Junto al cenador, en la esquina superior izquierda del plano, Petasite y en el cuadro cercano otras plantas de flor como Lilium martagon, prímulas, verónicas y ranúnculos, además de un cuadro dedicado al cultivo de fresas, una pila de agua que aparece en el plano junto al cenador, en la esquina superior izquierda, que contenían Menyanthes palustris y un depósito de general plantas. Le seguían 42 eras enfocadas al cultivo de plantas medicinales, una platabanda y 17 eras de plantas «sospechosas»: mandrágora, belladona, datura, beleño, cicuta, tejo, acónito, ricino y otras de carácter no tan venenoso.

Tras la descripción del contenido de los cuadros y eras, aparece la relación de los árboles y arbustos que bordean los caminos, destacando principalmente los olmos, robinias, jazmines, clemátides, granados, acacias, laureles, pistachos, acebos, castaños, avellanos, juniperus, etc. En los bordes de los cruceros de la cara norte hasta el estanque grande se hallaban plantadas diversas vides.

Otros elementos decorativos a los que se hace mención en el documento son la puerta de hierro que aparece en el plano en la cara opuesta a la del camino de El Pardo, el cenador a la izquierda y las casas del jardinero e invernáculo a la derecha

9 Biblioteca del Palacio de Santa Cruz de Valladolid, (S.C.M.), ms. 109. Gómez OrTegA, C. (1771), Inventario de todas las plantas, utensilios y enseres del Real Jardín Botánico, hecho en el mes de octubre de 177I; S.C.M., ms. 110, (1771, noviembre, 6), continuación del documento anterior. Madrid y S.C.M., ms. 111, (1771, noviembre, 15). Inventario de las semillas que se hallan y quedan en poder de la Sra. viuda del Dr. D. Miguel Barnades, como primer Profesor de Botánica que fue del Real Jardín Botánico. Madrid. 


\section{LA BOTÁNICA EN EL JARDÍN DE MIGAS CALIENTES}

del mismo lado, el estanque, la pila de agua junto al cenador, el estanquillo de las clases y el del emparrado.

\section{ENSEÑANZA DE LA BOTÁNICA}

La enseñanza de la botánica en el jardín de Migas Calientes dio comienzo en 1757 bajo la dirección de sus profesores, José Quer y Minuart: «Las Lecciones Botanicas, que todos los años publicamente se celebran por la Primavera; en cuya Estación manifiestan aquellos sabios Professores la perfección de la Botanica: enseñanza demostrativa, como lo acredita la concurrencia tan copiosa de Alumnos y estudiosos Discípulos, que en este tiempo de todas partes concurren» 10 .

Al ser Quer su principal profesor se puso gran énfasis en la importancia de la botánica aplicada, principalmente en su vertiente medicinal, eligiendo para su enseñanza el sistema tournefortiano por ser el de mayor calidad pedagógica. En 1764, tras el fallecimiento de Quer, le sucedía Miguel Barnades, educado en Montpellier e iniciado en los principios reformadores de Linneo, quien comenzó la introducción del método linneano sin alejarse en demasía de las antiguas prácticas ${ }^{11}$.

Durante los primeros años de enseñanza no se contó con ningún libro de texto. Quer intentó publicar sus Lecciones de Botánica, dictadas a uno de sus discípulos en 1762, pero quedaron manuscritas y tras haberse depositado por un tiempo en la Casa de Geografía, se trasladaron al Real Jardín Botánico de Madrid donde se conservan actualmente $^{12}$. Dichas lecciones están dispuestas según el método de Tournefort: cada una de las 22 clases, constituidas según la forma de la corola, está dividida en varias secciones, normalmente según las características del fruto, y éstas, a su vez, se escinden en diversos géneros de los que se mencionan sus usos medicinales.

Entre 1762 y 1764 comenzó la publicación de Flora Española, concluida veinte años después por Casimiro Gómez Ortega (1740-1818). Obra donde «se reduce á dar una Sencilla relación de quanto he visto, y observado en mis viages, y peregrinacio-

11) Quer, J. (1762), tomo I, pág. 31; en relación a este tema véase especialmente: PuIG SAMPER, M.A. (1987), opus cit., pp.60-63.

11 Colmeiro, M. (1875), pp.6-7.

12 Archivo del Real Jardín Botánico de Madrid, (R.J.B), div. 1, leg. 19, carp. 1. Quer, J. (1762), Praelectiones botanicae quibus plantarum et stirpium natura, virtutesque suculentissime describuntur, in Medicorum commodum et Pharmaceuticorum usum dictatae, A. D. Joseph Quer Primo regis hispaniarum botanico professore et ab Blassius E. Llamo, Minimo tanti viri Discipulo transcriptae. Anno 1762, 344 págs. Sobre su depósito en la Casa de Geografía véase: Corella Suárez, P. (1987), «La Real Casa de Geografía de la Corte y el comercio ultramarino durante el siglo XVIII», Anales del Instituto de Estudios Madrileños, tomo XXIV, p.224. 
nes por el Reyno en las Plantas» ${ }^{13}$ y en la cual las plantas aparecen ordenadas alfabéticamente para su uso como índice. Cada género se halla acompañado de la descripción dada por sus autores y de otra completa de su mano, más su correspondiente lámina; le sigue la descripción de la especie presente en España y la relación de sus principios químicos y usos medicinales. El primer tomo incluye igualmente una traducción comentada de la Isagoge (1700) de Tournefort, una relación histórica de los métodos aparecidos hasta el momento y la comparación de los sistemas tournefortiano y linneano.

No hay que negar que en el texto se aprecia el resentimiento de Quer por las críticas que lanzó Linneo acerca de la botánica española, pero las principales causas por las que rechaza su método son: 1) el «querer fundar el nervio, ó punto fundamental del dicho Méthodo por los estambres», demasiado pequeños a veces, despreciando el resto de los caracteres; 2) por las excepciones que Linneo debe realizar saltándose sus propios principios, lo que «manifiesta, que se ve las más veces forzado á seguir, y asi tacitamente á aprobar la sabia advertencia de Tournefort, que es: Que quales quiera Plantas, que convienen entre sí en la mayor parte de sus signos han de reducirse estos á un mismo género; aunque discrepen en algún signo leve», acusándole en otra parte de plagiar a Tournefort; 3) por la variabilidad del número de estambres; 4) por el uso del microscopio, que según Quer estaba prohibido por el Jardín Botánico de Padua, «no obstante, para hacer una exacta, y perfecta anatomia de algunas Plantas, confiesso, y tengo por conveniente el uso de estos opticos auxilios, sin los cuales sería impracticable»; 5) por el excesivo cambio de nombres, sin respeto a los autores antiguos; y 6) porque considera igualmente necesario introducir en la descripción el lugar de origen de cada planta, no simplemente para reconocer las mejores condiciones de su cultivo, sino porque «las especies de algunas Plantas, se conoce claramente, que son más que unas casuales diferencias, y bastardas variedades, que ocasionan la qualidad de los terrenos y climas. Assi, estas mismas especies que supone, colocadas en los kirrenos más propios de tales plantas, con el tiempo degeneran de tales variedades. y diferencias volviendose á la antigua naturaleza de las especies conocidas... Así. podemos conjeturar que se irán encontrando cada dia innumerables nuevas variedader de Plantas, que por expureas con el tiempo se desvaneceran; y podemos también juzgar ser esta la causa de que muchas Plantas, referidas por los antiguos hoy día no se encuentren» ${ }^{14}$.

Menos prolijo, el segundo profesor, Juan Minuart, publicó únicamente un par de disertaciones antes de ocupar su puesto en el jardín; en ellas se describen las plantas Cerviana y Cotyledon hispanica (1739).

13 QUER, J. (1962), tomo I, pp.1-2.

14 QUER, J. (1762), tomo I, pp.327-343. 
Por su parte, el sucesor de Quer, Miguel Barnades, publicó en 1767 Principios de Botánica, obra de la que había proyectado un segundo volumen destinado a exponer «todo lo que concierne al método de conocer clara, y distintamente las plantas, y nombrarlas con propiedad: propondré el Systema de su distribución en clases, ó familias, y ordenes, que creo el más fácil, y adaptado al común de los principiantes» ${ }^{15}$ Desgraciadamente este texto se quedó en el tintero, dejándonos sin saber cuáles fueron sus preferencias reales. Queda clara la fabulosa labor que realizó Linneo entre los reformadores de la botánica, principalmente en materia nomenclatural, pero las reformas no pararon allí, otros muchos habían contribuido a «mejorar la ordenación de las plantas, dejando la artificial o Systematica, y tanteando la más conforme á la Naturaleza, que también trazó Linneo en los que intituló Fragmentos del método natural» ${ }^{16}$, destacando, por ser los más recientes y dignos del mayor aprecio, Michel Adanson (1727-1806) y el botánico y médico alemán Oeder (1728-1791).

Al primero y a su obra, Familles des plantes (1763), dirige su reconocimiento: «La ingenuidad sobre los defectos que reconoce este autor hasta en su proprio plan metodico, la exactitud con que cumple lo que promete, y la claridad en sus expresiones, pueden servir de modelo á los mas clasicos escritores de Botanica y la execucion de sus consejos podría encaminar esta ciencia á un grado de certeza de que dista mucho por ahora» ${ }^{17}$.

Por todo ello, quizá habría que matizar la catalogación de Barnades como estricto linneano, ya que claramente se muestra favorable a la adopción de un método natural del que puedan inferirse la cualidades medicinales de las plantas, razón de ser de la botánica: «Mas la botánica enseña el conocimiento de las virtudes á lo menos generales de las plantas por medio del estudio de las afinidades naturales...Sabiendose por la Botanica la familia ó genero natural á que pertenece, se podrá asegurar las virtudes más principales que se saben por la experiencia de las demás de su clase ó género natural» ${ }^{18}$.

Sobre el resto de su obra, Colmeiro nos dice que su Muestra de la Flora Española (Specimen Flora hispanica), manuscrito acompañado de dibujos que fue aumentado por su hijo, quedó también inédito conservándose únicamente la parte iconográfica $^{19}$.

\footnotetext{
15 Barnades, M. (1767), Principios de Botánica, Madrid, «Al lector» s.p.

16 Ibidem, p. 19.

17 BARNADES, M. (1767), p.21. Véase en relación a este tema: Puig SAMPER, M.A. (1987), pp.6162.

18 BARNADES, M. (1767), p.35.

19 Tal vez se corresponda con la obra iconográfica en 6 volúmenes, que se conserva en el Real Jardín Botánico, bajo el título de Herbarium pictum, atribuido por Lagasca a Miguel Barnades en una carta que acompaña a esta colección de láminas.
} 
Indiscutiblemente, el cambio de metodología en la concepción del jardín vino de la mano de Gómez Ortega, sucesor de Barnades a su muerte en 1771. A sus primeras obras, Comentario o Tratado de la Cicuta (1763), descripciones de Cotyledon Mucizonia y Pistorinia (1772), le seguirían: Tabula botanica tournefortiance (1773), Catálogo de las plantas que se crian en el sitio de los baños de Trillo (1778); Instrucción sobre el modo más seguro y económico de transportar plantas vivas (1779); Historia natural de Malagueta (1780).

Se ha señalado que la adhesión de Ortega al método linneano se debió a motivos pragmáticos más que filosóficos; transición lenta que durante los primeros años condujo a la traducción del sistema de Tournefort, puente con la tradición del antiguo jardín ${ }^{20}$.

El traslado del jardín botánico desde el Huerto de Migas Calientes hasta su actual emplazamiento en el Prado de Atocha, marcó el arranque del nuevo sistema teórico en la enseñanza de la botánica, para lo cual Antonio Palau y Verdera publicó Explicación de la Filosofía y Fundamentos botánicos de Linneo $(1778)^{21}$.

\section{ALGUNOS COMENTARIOS SOBRE LOS BOTÁNICOS DEL JARDÍN}

«Los botánicos, aquí en Madrid, son hombres interesantes, aunque casi demasiado fieles a Tournefort» ${ }^{22}$, así describía Pehr Löfling (1729-1756), discípulo de Linneo que llegó a España en 1751, a la plantilla de botánicos que constituirían el nuevo Jardín de Migas Calientes. Minuart, educado en Francia y discípulo del botánico Jaime Salvador, seguía el método del francés y aunque sintiera cierto interés por conocer el linneano, por estar basado en caracteres a veces difícilmente observables como los estambres, le imposibilitaba su utilización por su menguada visión; el joven Cristóbal Vélez se mostraba más inclinado a su uso por la nitidez de los rasgos; a pesar de ello su obra Flora Matritensis fue escrita según el método de Tournefort. Sin duda el más recalcitrante al cambio fue Quer.

20) Puerto Sarmiento, J. \& GonzÁlez Bueno, A. (1987), «Renovación sanitaria y utilidad comercial: las expediciones botánicas en la España ilustrada», Revista de Indias, XLVIII, 180, p.491; y GONZÁlez BuENo, A. \& Ruiz OCHAITA (1985), «La introducción de la filosofía linneana en la Botánica española, actitud de Gómez Ortega (1748-1818)», Bol. Soc. Esp. Hist. Farm. 34, pp.141-142.

21 Colmeiro, M. (1875), p.14.

22 F. Pelayo (ed.) (1990), «Cartas inéditas en español de Pehr Löflin», en Pehr Löfling y la Expedición al Orinoco, 1754-1761, Colección encuentros, Real Jardín Botánico, C.S.I.C.-Quinto Centenario, Madrid, p.125. 
José Quer nació en Perpignán el 26 de enero de 1695 y murió en Madrid el 19 de marzo de $1764^{23}$. En su ciudad natal cursó los primeros estudios de medicina; pronto obtuvo el puesto de cirujano en el Regimiento de Soria, por entonces de guarnición en Gerona, junto al que recorrió y herborizó en 1729 las provincias de Cataluña, Aragón y Valencia. En 1732 pasó a Orán con el deseo de explorar las costas africanas. A su vuelta a España residiría algún tiempo en Alicante, colectando en Valencia y la provincia de Murcia. En 1733 viajó a Italia junto a su regimiento, estancia que aprovechó para herborizar y mantener frecuentes contactos con el Jardín Botánico de Pisa. En 1737 vuelve a Barcelona y de ahí a Madrid donde instala un pequeño jardín botánico para el cultivo de aquellas plantas recogidas en los alrededores en compañía de Minuart y Vélez. En 1741 fue nombrado Cirujano Consultor de la Armada, embarcándose ese mismo año de nuevo hacia Italia. En Bolonia siguiría las lecciones de Monti y con Minuart exploró los alrededores de Nápoles y Roma. En 1745 regresa a España vía Montpellier, donde entra en contacto con Sauvages y en Barcelona visita Montserrrat. Desde 1746 se dedica al cultivo de especies alimenticias en el jardín del duc d'Atrisco. Entre 1749 y 1752 exploró la sierra de Extremadura, la laguna de Gredos y los montes de Ávila. Tras la creación del Jardín Botánico del Soto de Migas Calientes, Quer es nombrado primer profesor.

En 1762 salían a la luz los tres primeros volúmenes de Flora de España, a los que siguió un cuarto en 1764 . Veinte años después de la muerte del autor, y gracias a la colaboración de Gómez Ortega, se publicaban los volúmenes quinto y sexto. Su obra es un compendio de sus primeras herborizaciones por la Península, continuadas en la primavera de 1761 por las montañas de Burgos, León, Asturias y Galicia ${ }^{24}$, a las que unió los materiales del herbario de Vélez y el manuscrito Flora matritensis de este mismo botánico, inédito desde 1753.

Durante su dirección, el jardín entró en relaciones con Bolonia, París, Leiden y Amsterdam, enriqueciendo su herbario con plantas de esos países y de España a través de los correspondientes del jardín, así como de la América española y las colonias inglesas, llegando a contar con un total aproximado de 2.000 especies dispuestas según el sistema tournefortiano.

23. Gómez OrTega, C. (1784), «Elogio histórico de Don Joseph Quer», en Quer, J., Flora Española, Madrid, vol. V, pág. 32. Colmeiro, M. (1858), La botánica y los botánicos de la Península Hispano-Lusitana, Madrid, pp.163-165. BRIQUET, J. (1919), «Les collections botaniques du botaniste espagnol José Quer, (1695-1764)», Annuaire du conservatoire et du jardin botaniques de Genéve, XX, pp.465-478. PASCUAL, R. (1970), «El botánico José Quer (1695-1764) primer apologista de la ciencia española», Cuadernos Valencianos de Historia de la Medicina y de la Ciencia, ser. B, $\mathrm{n}^{\circ}$ 10, pp. 1-82.

24 Sobre estas últimas herborizaciones puede consultarse el intinerario en GARCÍA GUARDIA, G. (1986), «Itinerario de José Quer en su expedición botánica de 1761», en: P. MARTín FerRero (ed.), Actas del simposium CCL aniversario nacimiento de Joseph Celestino Mutis, Diputación Provincial de Cádiz, Cádiz, pp. 215-223 y las peticiones de ayuda económica en: Añón, C. (1986), pp.30-32. 
En relación a la biblioteca del jardín, en 1781 tenía unos 250 volúmenes, sobrepasando pocos años después los 1.000 ejemplares al incorporarse a ella los 849 títulos sobre materia médica, historia natural y botánica que Quer tenía en propiedad ${ }^{25}$.

Con una imagen un tanto desprestigiada, se le ha achacado el desconocimiento de las corrientes botánicas de su tiempo, idea que parece contraria a quien posee 166 volúmenes únicamente de botánica, entre los que se cuentan no sólo la mayoría de los autores antiguos, sino también los clásicos en su época como son Ray, Morison, Magnol, por supuesto Tournefort y 12 de las obras de Linneo: Bibliotheca botanica (1736), Hortus Cliffortianus (1737), Flora Lapponica (1737), Classes plantarum (1738), Oratio de necessitate peregrinationum (1743), Flora Suecica (1745), Flora Zeylanica (1747), Hortus Upsaliensis (1748), Materia medica I (1749), Amoenitates academicae (1751).

25. COlmeiro, M. (1875), pp.6-7. 


\section{APÉNDICE}

\section{BIBLIOTECA BOTÁNICA DE JOSÉ QUER}

El inventario de los volúmenes dedicados a la botánica que pertenecieron a la biblioteca de Quer aparece como apéndice documental a este artículo ${ }^{26}$. A la indicación dada en el documento de cesión le sigue la identificación del volumen -a veces de una forma aproximada-, en términos de autor, nombre de la obra, localidad y fecha.

Libros de botánica y ciencias afines

1) 2.tomos En folio y Pasta Roberto, Morison Historia Plantarum, en Oxonii año de 1715.

Morison, Robert

Plantarum historiae universalis Oxoniensis seu herbarum distributio nova, per tabulas cognationis \& affinitatis ex libro naturae observata \& detecta / auctore Roberto Morison... Oxonii: e Theatro Sheldoniano; et prostant Londini: apud Paulum \& Isaacum Vaillant, 1715,3 tomos en 2 volúmenes ( $1^{\mathrm{a}}$ ed., Oxford, 1672,1680 y 1699 ).

2) 1.tomo En folio y Pasta Joanis, Commelino Horti Medici en Ansterdami año de 1697.

Commelin, Jan

Horti medici Amstelodamensis rariorum tam Orientalis, quam Occidentalis Indiae,... auctore Joanne Commelino...

Amstelodami: apud P.\& J. Blaeu, nec non Abrahamum a Someren, 1697.

26 R.J.B., Div. 1, leg. 4, carp. 5, doc. 11, (1787, agosto, 30-1787, septiembre, 15). Catálogo de los libros de J. Quer trasladados al R.J.B. El apéndice que acompaña a este trabajo está basado en este documento.

El resto de los volúmenes de medicina y otras materias fueron trasladados al Colegio de Cirugía y a la Secretaría de Indias, véase: R.J.B., Div. 1, leg. 3, carpt. 2, doc. 2., (1781, agosto, 20) Inventario de los libros de botánica, de química y de historia natural existentes en la librería del Real Jardín Botánico; R.J.B., Div. 1, leg. 4, carpt. 5, doc. 10., (1787, diciembre, 18). Real orden para que se pasen al Colegio de Cirugía los libro de J. Quer referentes a medicina, cirugía y anatomía, firmada por A. Gimbernat; R.J.B., Div. 1, leg. 4, carpt. 7, doc. 8., (1788, abril, 27). Lista de los libros restantes de la biblioteca de J. Quer, después de haberse separado los de botánica para el uso del Real Jardín Botánico y los que se han entregado al Colegio de Cirujanos; R.J.B., Div. 1, leg. 4, carpt. 7, doc. 9., (1788, julio, 1). Catálogo de los libros que fueron de J. Quer y se han separado de los de botánica e historia natural y se han entregado en la Secretaría de Indias; R.J.B., Div. 1, leg. 4, carpt. 7, doc. 10., (1788). Lista de los libros restantes de la biblioteca de J. Quer después de haberse separado los de botánica y química. 
3) 1.tomo En folio y Pergamino Lobeli Stirpium Historia en Antuerpie año de 1576.

L'Obel, Matthias de

Plantarum seu stirpium historia,...

Antverpiae: ex officina Christophori Plantini architypographi regii, 1576.

4) 1.tomo En Pasta cuarta ynperial Lobeli Icones Plantarum en Antuernie año de 1591.

L'Obel, Matthias de

Icones stirpium,...

Antverpiae: ex officina Plantiniana, apud viduam et Ioannem Moretum, 1591. (1 1 $^{\mathrm{a}} \mathrm{ed} .$, Anvers, 1581).

5) 1.tomo En Pasta Octavo Flora Malabarica, Commelin en Ludumi año de 1696.

Commelin, Caspar

Flora Malabarica, sive Horti Malabarici catalogus exhibens omnium ejusdem plantarum nomina...

Lugduni Batavorum: apud Fredericum Haaringh, bibliopolam, 1696.

6) 1.tomo En quarto y Pasta Caroli Allioni, Societate Physica Botanica en Augusta año de 1755.

Allioni, Carlo

¿Rariorum Pedemontii stirpium specimen primum?

Augusta Taurinorum, 1755.

7) 1.tomo En Pasta quarto ymperial Commelino, Plantarum rariorum orti Medici en Luduni año de 1706.

Commelin, Caspar

Horti medici Amstelaedamensis plantae rariores et exoticae ad vivum aeri incisae.

Leiden (Lugdunum Batavorum), 1706.

8) 3.tomos En folio y Pasta Ray, Historia Plantarum en Loduni año de 1686.

Ray, John

Historia plantarum...

Londini: typis Mariae Clark: prostant apud Henricum Faithorne \& Joannem Kersey ad insigne Rosae in coemeterio D.Pauli, \& e regione aedium Bedfordiensium in vico the Strand dicto, 1686- 1688. (tomo III, 1704).

9) 1.tomo En folio y Pasta ynperial, Schewzero, Herbarium Dilubianum en Luduni año de 1723.

Scheuchzer, Johann Jacob

Herbarium diluvianum

Lugduni Batavorum: sumptibus Petri Vander Aa, bibliopolae, civitatis atque academiae typographi, 1723. ( ${ }^{\text {a }}$ ed. en Zurich, 1709)

10) 1.Biblioteca Botanica en folio $4^{\circ}$ en Pasta en hagae año de 1740.

Seguier, Jean François 


\section{LA BOTÁNICA EN EL JARDÍN DE MIGAS CALIENTES}

Bibliotheca botanica,...

Hagae-Comitum: apud Joannem Neaulme, 1740.

11) 1.Caroli Linei Oratio de necessitate peregrinatior un tomo en octabo y pasta en Luduni año de 1743 .

Linné, Carl von

Oratio de necessitate peregrinationum...

Lugduni Batavorum: apud Cornelium Haak, 1743.

12) Biblioteca Botanica Caroli Linei un tomo en octabo y Pasta en Asterdan año de 1736. Linné, Carl von

Bibliotheca botanica

Amstelodami: apud Salomonem Schouten, 1736.

13) De Amoenitates Academie Linei un tomo en octabo y Pasta en Horme año de 1751. Linné, Carl von Amoenitates academicae,...

14) Materia Medica Linei un tomo en octabo y Pasta en Horme año de 1749.

Linné, Carl von

Materia medica, liber I.

Holmiae: typis ac sumptibus Laurentii Salvii, 1749.

15) Linei Hortus upsaliensis un tomo en octabo y Pasta en Stockholmie año de 1748.

Linné, Carl von

Hortus Upsaliensis

Stockholmiae: sumtu \& literis Laurentii Salvii, 1748.

16) . Caroli Linei Critica Botanica un tomo en $4^{\circ}$ y Pasta en Luduni año de 1737.

Linné, Carl von

Critica botanica

Lugduni Batavorum: apud Conradum Wishoff, 1737.

17) Linei Clases Plantarum un tomo en octabo y Pasta en Luduni año de 1738

Linné, Carl von

Classes plantarum

Lugduni Batavorum: apud Conradum Wishoff, 1738.

18) Linei Flora Lamponica un tomo en octabo y Pasta en Asterdan año de 1737.

Linné, Carl von

Flora Lapponica

Amstelaedami: apud Salomonem Schouten, 1737.

19) Linei Amonetatis Academice un tomo en octabo y Pasta en Holmie año de 1749.

Linné, Carl von 


\section{SUSANA PINAR Y MIGUEL ANGEL PUIG-SAMPER}

Amoenitates academicae.., volumen primum.

Lugduni Batavorum: apud Cornelium Haak, 1749.

20) Linei Flora Suecica un tomo en octabo y Pasta en Luduni año de 1745.

Linné, Carl von

Flora Suecica

Lugduni Batavorum: apud Conradum Wishoff \& (apud) Georg. Jac. Wishoff, fil. Conr., 1745 .

21) Linei Flora Zeylanica un tomo en octabo y Pasta en Holmie año de 1747.

Linné, Carl von

Flora Zeylanica

Holmiae: sumtu \& literis Laurentii Salvii, 1747.

22) Fragoso de Aromas de Indias un tomo en octabo y Pergamino en Madrid año de 1572.

Fragoso, Juan

Discursos de las cosas aromaticas, arboles y' frutales, y de otras muchas medicinas simples que se traen de la India Oriental, y sirven al uso de medicina...

Madrid: Impresso en casa de Francisco Sanchez, vendense en casa de Sebastian Yuañez, 1572 .

23) (Grew) Anatomia de Plante un tomo en octabo y Pergamino (en italiano) en Venecia año de 1763.

Grew, Nehemiah

Posible traducción de The Anatomy of Plants.

London, 1682.

o traducción de Anatomie des plantes..

Paris, 1675.

24) Ray Joannis, (Methodus) Plantarum Joanis un tomo en $4^{\circ}$ y Bitela en Londini año de 1703.

Ray, John

Methodus plantarum

Londini: impensis Samuelis Smith \& Benjamini Walford typographorum Regiae societatis, ad insignia principis in coemeterio D. Pauli.Et veneunt Amstelaedami: apud JanssonioWaasbergios, 1703. ( $1^{\text {a }}$ ed. en Londres, 1682)

25) Raij Sinopsis Stirpium Britanica un tomo en octabo y Pasta en Londini año de 1724.

Ray, John

Synopsis methodica stirpium Britannicarum

Londini: impensis Gulielmi \& Joannis Innys Regiae societatis typographorum, in Area occidentali D. Pauli, 1724, $3^{\mathrm{a}}$ ed. ( $\mathrm{l}^{\mathrm{a}}$ ed. en Londres, 1690)

26) Suppmto. Adabregè de Hist ${ }^{\text {a }}$.de Plantes tres tomos en octabo y Bitela en Paris año de 1730.

27) Ammanis Stirpium Rariorum un $\mathrm{t}^{\circ}$ en Cuarto y Pasta en Petropoli. Ammann, Johann 


\section{LA BOTÁNICA EN EL JARDÍN DE MIGAS CALIENTES}

Stirpium rariorum in Imperio Rutheno sponte provenientium icones et descriptiones.

Petropoli: ex typographia Academiae scientiarum, 1739.

28) Mapi Hist ${ }^{\mathrm{a}}$ Plantarum un tomo en Cuarto y Pasta en Asterdan año de 1742.

Mappus, Marcus

Historia plantarum Alsaticarum

Amstelodami: apud Petrum Mortier bibliop., 1742.

29) Bonnet (Recherches sur) des fobilles un tomo en Cuarto y Pasta en Agotingue año de 1754.

Bonnet, Charles

Recherches sur l'usage des feuilles dans les plantes

Göttingen, 1754.

30) Metodus foliorum un tomo en Cuarto y Pasta en Alahae año de 1751

Boissier de Sauvages, François

Methodus foliorum

A La Haye: (s.n.), 1751.

31) Ximenez Plantas Animales de Indias, un tomo en Cuarto y Pergamino en Mexico año de 1615.

Ximénez, Francisco

Quatro libros de la Naturaleza y virtudes de las plantas, y animales que están recevidas en el uso de Medicina de la Nueva España,...

En México: Casa de la viuda de Diego Lopez Davalos, 1615.

32) Monardis de drogas de Indias un tomo en octabo y Pergamino en Sevilla año de 1569.

Monardes, Nicolás

Dos libros. El uno trata de todas las cosas que traen de nuestras Indias Occidentales que sirven al uso de medicina...El otro libro, trata de dos medicinas maravillosas que son contra todo veneno, la piedra bezaar, y la yerba escuerçonera...

Sevilla: Casa de Hernando Diaz, 1569. (1 ${ }^{\mathrm{a}}$ ed. en Sevilla, 1565)

33) Gmelin Noborum Begetabilium un tomo en octabo y Pasta.

Gmelin, Johann Georg

Sermo de novorum vegetabilium exortu.

Tubingae: literis Erhardtianis, [1749].

34) Statique de Begetaux et des Animaux dos tomos en Cuarto y Pasta en Paris el uno año de 1735 y el otro en genova año 1744

Hales, Stephen

La statique des végétaux

A Paris: chez Debure l'aîné, à l'entrée du quay des Augustins, du côté du pont Saint Michel, à Saint Paul, 1735.

35) Escheocero Historia Graminum un tomo en cuarto y Pasta en Tiguri año de 1719.

Scheuchzer, Johann 
Agrostographia sive graminum, juncorum, cyperorum, cyperoidum, iisque affinium historia.

Tiguri: typis \& sumptibus Bodmerianis, 1719.

36) Paulli Cuadripartitum Botanicum un tomo en Cuarto y Pasta en Franconforti.

Paulli, Simon

Quadripartitum botanicum

Francofurti: apud Georgium Henricum Oehrlingium, 1708. ( $1^{\mathrm{a}}$ ed. en Rostock, 1639, $2^{\mathrm{a}}$ ed. en Estrasburgo, 1667-68)

37) Relacion de un voyage de Tournefort tres tomos en Cuarta y Pasta en Leon año de 1727.

Tournefort, Joseph Pitton de

Relation d'un voyage du Levant

A Lyon: chez les frères Bruyset, 1717.

38) Histoire de Plantes de París Mons. Piton dos tomos en octabo y Pasta en Paris año de 1725. Tournefort, Joseph Pitton de

Histoire des plantes qui naissent aux environs de Paris

Paris: chez Jean Musier, quai des Augustins, du côté du Pont S.Michel, à l'Olivier, 1725, $2^{\mathrm{a}}$ ed.

39) Traite des Abres et Arbustes dos tomos en Cuarto y Pasta en Paris año de 1755.

Duhamel de Monceau

Traité des Arbres et Arbustes qui se cultivent en France en pleine terre

Paris, 1755, 2 vols.

40) Histoire de Plantes Memoires un tomo en 4 y Pasta en Amsterdan año de 1758.

4.1) Malpighii Anatome Plantarum en tres tomos en folio y Pasta en Londine año de 1675 y otros años.

Malpighi, Marcello

Anatome plantarum

Londini: impensis Joannis Martyn, Regiae societatis typographi, ad insigne Campanae in coemeterio Divi Pauli, 1675-1679.

42) Historia Ex Plantei Generale Dos tomos en folio y Pasta en Alion año de 1653.

Dalechamps, Jacques

Histoire générale des plantes

A Lyon: chez Philip. Borde, Laur. Arnaud \& Cl. Rigaud, 1653. (1ª ed. en Lyon, 1615)

43) Histoire de Plantes Quinaisen de la Probence un tomo en folio y Pasta en Aix año de 1715. Garidel, Pierre Joseph

Histoire des plantes qui naissent aux environs d'Aix, et dans plusieurs autres endroits de la Provence.

A Aix: chez Joseph David, imprimeur du roy, du pays \& de la ville, au Roy David, 1715. 


\section{LA BOTÁNICA EN EL JARDÍN DE MIGAS CALIENTES}

44) Micheli Catalogus florentino un tomo en $4^{\circ}$ y Pasta en Florencia año de 1748.

Micheli, Pier Antonio

Catalogus plantarum Horti caesarei Florentini

Florentiae: ex typographia Bernardi Paperinii, 1748.

45) Aldino Hortus Farnesianus un tomo en folio y encerado berde en Roma año de 1625.

Aldinus, Tobias

Exactissima descriptio rariorum quarundam plantarum, quae continentur Romae in Horto

Farnesiano.

Romae: typis Iacobi Mascardi, 1625.

46) Hortus Catolicus un tomo en $4^{\circ}$ y Pergamino en Napoles año de 1696.

Cupani, Francesco

Hortus catholicus

Neapoli: apud Franciscum Benzi, 1696.

47) Dodonei Stirpium histori un tomo en folio y Bitela en Antuerpie año de 1616.

Dodoens, Rembert

Stirpium historiae pemptades sex sive libri XXX

Antverpiae: ex officina Plantiniana apud Balthasarem et Ioannem Moretos, 1616. ( $1^{\circ} \mathrm{ed}$. en Anvers, 1583)

48) Traité de la culture des Renuncules un tomo en $4^{\circ}$ y pasta en Paris año de 1754.

(Podría ser una edición del libro de Jean Paul de Rome d'Ardene)

49) Allerus opuscula Botanica un tomo en $4^{\circ}$ y pasta en Gotingae año de 1749.

Haller, Albrecht von

Opuscula botanica

Gottingae: apud Io. Wilh. Schmid., acad. bibliop., 1749.

50) Diccionario Botanico ex Farmaceutique un tomo en $4^{\circ}$ y pasta en Paris año de 1748.

51) Ludobique Difiniciones Generum Plantarum un tomo en $4^{\circ}$ y pasta en Lidsiae año de 1747. Ludwig, Christian Gottlieb

Definitiones generum plantarum

Lipsiae: apud Iohann. Frideric. Gleditschium, 1747.

52) Nuebo Discurso de la Generacion de las Plantas e ynsectos un tomo en $4^{\circ} \mathrm{A}$ la Rustica en Madrid año de 1747.

García Hernández, Francisco

Nuevo discurso de la generación de las plantas, insectos, hombres y animales

Madrid, 1747.

53) Escopoli Flora Carneolica Joanis Antoni escopoli un tomo en $4^{\circ}$ y pasta en Bienae año de 1760.

Scopoli, Giovanni Antonio 


\section{SUSANA PINAR Y MIGUEL ANGEL PUIG-SAMPER}

Flora Carniolica

Vienna, 1760

54) Gaspari comelino prelutia Botanica un tomo en $4^{\circ}$ y pasta en Luduni año de 1703.

Commelin, Caspar

Praeludia botanica

Lugduni Batavorum: apud Fredericum Haringh, 1703.

55) Jungii opuscula Botanica Phisica un tomo en $4^{\circ}$ y pasta en Courgui año de 1747.

Jung, Joachim

Opuscula botanico-physica

Coburgui: sumtibus et typis Georgii Ottonis, typogr. ducal. priv., 1747.

56) Noba Plantarum Amedicanarum Genera un tomo en $4^{\circ}$ y pasta en París año de 1703.

Plumier, Charles

Nova plantarum Americanarum genera

Parisiis: apud Ioannem Boudot, Regis \& Regiae scientarum academiae typographum, via Jacobaea, ad Solem Aureum, 1703.

57) Petri Magnol Medici Nobus caracter Plantarum un tomo en $4^{\circ}$ y pasta en Monspellii año de 1720

Magnol, Pierre

Novus caracter plantarum

Monspellii: apud viduam Honorati Pech..., 1720.

58) Maneti Biridarium Florentinum un tomo en $4^{\circ}$ y pasta en Florencia año de 1751

Manetti, Xaviero

Viridarium Florentinum

Florentiae: ex typographia Bernardi Paperini, 1751.

59) Flora Ferari Josef Batiste un tomo en $4^{\circ}$ y Pergamino en Asterdami año de 1646. Ferrari, Giovanni Battista

Flora

Amstelodami: prostant apud Joannem Janssonium, 1646. (1 ${ }^{\mathrm{a}}$ ed. en Rome, 1633)

60) Elemento de Botanico de Abrefer un tomo en octabo y Pasta en Abicnon año de 1749.

Tournefort, Joseph Pitton de

Abrégé des "Elémens de Botanique ou méthode pour connaître les plantes.

Avignon, 1749.

61) Boehmeri Flora Lipsie yndigena un tomo en $4^{\circ}$ y Pasta en Lisiae año de 1750.

Boehmer, Georg Rudolf

Flora Lipsiae indigena

Lipsiae: apud Ioh. Gothofred. Dyckium, 1750. 


\section{LA BOTÁNICA EN EL JARDÍN DE MIGAS CALIENTES}

62) Orta Drogas de Indias Aromatum et simplicium aliquor un tomo en $4^{\circ}$ y pergamino en Antuerpie año de 1579 .

Orta, Garcia da

Aromatum et simplicium... historia

Antverpiae: ex officina Christophori Plantini, 1579, $3^{\mathrm{a}}$ ed. ( $1^{\mathrm{a}}$ ed. en Anvers, 1567 y ed.II en Anvers, 1574)

63) Institucion Rey erbari Josefi piton tres tomos en $4^{\circ}$ y Pasta en Paris año de 1700.

Tournefort, Joseph Pitton de

Institutiones rei herbariae

Parisiis: e typographia regia, 1700.

64) Columna de Plante Autore Fabeo Columnea un tomo en $4^{\circ}$ y Pasta en Napoles año de 1592.

Colonna, Fabio

Pytobasanos sive plantarum aliquot historia

Neapoli: ex officina Horatii Salviani, 1592.

65) Vaillant Sermo de structura Florum un tomo en $4^{\circ}$ y Pasta en Luduni año de 1718.

Vaillant, Sébastien

Sermo de structura florum

Lugduni Batavorum: apud Petrum Vander Aa...,1718.

66) Monardes cosas de Indias un tomo en $4^{\circ}$ y Pergamino en Sevilla año de 1580.

Monardes, Nicolás Bautista

Primera y segunda y tercera partes de la Historia Medicinal de las cosas que se traen de nuestras Indias Occidentales,...

Sevilla: Casa de Fernando Diaz, 1580.

67) Historia Medica de Indias un tomo en $4^{\circ}$ y Pergamino en Sevilla año de 1574.

Monardes Historia de Indias un tomo en $4^{\circ}$ y Pergamino en Sevilla año de 1574.

Monardes, Nicolás Bautista

Primera y segunda y tercera partes de la historia medicinal de las cosas que se traen de nuestras Indias occidentales...

Sevilla: Alonso Escribano, 1574, $1^{a}$ ed.

68) Tratado de las drogas y Medicinas de las Indias Orientales un tomo en $4^{\circ}$ y Pergamino en Burgos año de 1578.

Acosta, Christoval

Tractado de las drogas, y medicinas de las Indias Orientales,...

En Burgos: por Martin de Victoria impresor de su Magestad, 1578.

69) Clusi Stirpium espanie un tomo en $4^{\circ}$ y Pasta en Antuerpia año de 1576.

L'Escluse, Charles de

Rariorum aliquot stirpium per Hispanias observatarum historia

Antverpiae: ex officina Christophori Plantini, 1576. 


\section{SUSANA PINAR Y MIGUEL ANGEL PUIG-SAMPER}

70) Plantarum efigies et similitudinis que dibersis linguis redite un tomo en octabo y Pasta descuadernado en Luduni año de 1613.

71) Gaspari bauini basit un tomo en $4^{\circ}$ y Pergamino en Bisileae año de 1613. Posiblemente De lapidis bezaar, Basilea, 1613, 1ª ed.

72) Plantarum rariorum ex soticarum inorto Kleniniar un tomo en $4^{\circ}$ y Pergamino en Gedani año de 1748 .

(Puede ser obra de Breyn)

73) Tilli Plantarum orti pisani en florencia año de 1723 en folio y Bitela.

Tilli, Michelangelo

Catalogus plantarum Horti Pisani

Florentiae: typis Regiae Celsitudinis: apud Tartinium \& Franchium, 1723.

74) Zanoni Radiorum stirpium en Bolonia año de 1742 en folio y Bitela.

Zanoni, Giacomo

Rariorum stirpium historia

Bononiae: ex typographia Laelii a Vulpe, 1742.

75) Barrelier Plante año de 1714 en folio y Pasta.

Barrelier, Père Jacques

Plantae per Galliam, Hispaniam et Italiam observatae

Parisiis: apud Stephanum Ganeau, 1714.

76) Haller enumeracium Plantarum año de 1742 en folio y Pasta.

Haller, Albrecht von

Enumeratio methodica stirpium Helvetiae indigenarum

Gottingae: ex officina academica Abrami Vandenhoek, 1742.

77) Ermanie Materie medice año de 1726 un tomo en $4^{\circ}$ y Bitela en Argentina.

Hermann, Paul

Cynosura materiae medicae

Argentorati: sumptibus Johannis Beckii, 1726.

(En la lista se incluyen igualmente ediciones de 1729, 1731 y 1745)

78) Muntingi bera curture Plantarum en Asterdan año de 1672 un tomo en $4^{\circ}$ y Pergamino.

Munting, Abraham

Waare oeffening der planten

T'Amsterdam: voor Jan Rieuwertsz, 1672.

79) Caroli clusio Atrebatis exoticorum año de 1605 un tomo en folio y Pergamino.

L'Escluse, Charles de

Exoticorum libri decem

[Anvers]: ex officina Plantiniana Raphelengii, 1605. 


\section{LA BOTÁNICA EN EL JARDÍN DE MIGAS CALIENTES}

80) Bauini teatri Botanici en Basilea año de 1658 en folio y Bitela.

Bauhin, Caspar

Theatri botanici

Basileae: apud Ioannem Köning, 1658.

81) Zanicheli Historia de leplante en Belecia año de 1735 en folio y Bitela.

Zannichelli, Gian Girolamo

Istoria delle piante

In Venezia: appresso Antonio Bortoli, 1735.

82) Muntigui Fitografia curiosa en Asteledami año de 1727 en folio y Pasta.

Munting, Abraham

Phytographia curiosa

Amstelaedami: apud Franciscum Halmam, 1702. (Podría ser otra edición)

83) Tournefor Rey Herbari en Paris año de 1719 en tres tomos en $4^{\circ}$ y Pasta.

Tournefort, Joseph Pitton de

Institutiones rei herbariae

Parisiis: e typographia regia, $1719,3^{\mathrm{a}}$ ed.

84) Morandi Historia Botanica en Medioladi año de 1744, un tomo en Folio y Pasta.

Morandi, Giovanni Battista

Historia botanica pratica

Mediolani: ex typographia Petri Francisci Malatestae, 1744.

85) Aromati dela yndia en Benecia año de 1589 un tomo en $4^{\circ}$ y Filete encarnado y dorado.

Orta, Garcia da

Dell'historia de i semplici aromati, et altre cose...

Venetia: Li Heredi di Francesco Ziletti, 1589.

86) Florae Luduno Batabe flores en Leon año de 1690 un tomo en $4^{\circ}$ y Bitela.

Hermann, Paul

Florae Lugduno-Batavae flores

Lugd.Batavor.: apud Fredericum Haaring, 1690.

87) Ambrosini Ortus estudiosorum Bonie consitur en el año de 1657 un tomo en $4^{\circ}$ y Pergami-

no.

Ambrosini, Giacinto

Hortus Studiosorum

Bononiae: Typis Jo. Baptistae Ferroni, 1657.

88) Grisley Viridarium Lusitanum ynquo un tomo en $4^{\circ}$ y Bitela.

Grisley, Gabriel

Viridarium Lusitanum

Ulyssipone: ex praelo Antonii Craesbeeck, 1661. 
89) Tita Catalagus Plantarum en Patabi año de 1713 un tomo en $4^{\circ}$ y pergamino.

Tita, Antonio

Catalogus plantarum, quibus consitus est Patavii amoenissimus hortus...

Patavii: typis Semin.: apud Joannem Manfre, 1713.

90) Boeraave Index Plantarum orto Lodono batabi año de 1710 en Leon un tomo en $4^{\circ}$ y Pergamino.

Boerhaave, Herman

Index alter plantarum

Lugduni Batavorum: apud Janssonios Vander Aa, 1727. (1 $1^{\mathrm{a}}$ ed. en Leyden, 1720)

91) Pauli Renealmi Blaesensis en Paris año de 1611 en $4^{\circ}$ y Bitela.

Reneaulme, Paul II de

Specimen historiae plantarum

Parisiis: apud Hadrianum Beys, 1611.

92) Agricultura de Mr.Jobani tati en Benecia año de 1561 un tomo en $4^{\circ}$ y Bitela.

Tatti, Giovanni

Della Agricoltura

In Venetia: appresso F. Sansovino, 1561.

93) Marsili de Fungis Disertacion de Generacion, año de 1714 un tomo en Folio en Roma y Pasta.

Marsigli, Luigi Ferdinando

De generatione fungorum

Romae: ex officina typographica Francisci Gonzagae, 1714.

94) Theophrasto Ystoria Plantarum en Asterdan año de 1744 dos tomos en folio y Pasta.

Teofrasto

De historia plantarum

Amstelodami: apud Henricum Laurentium, 1644. (¿Otra edición?)

95) Thournefort contractus suformae tabula aum.en Franfort año de 1715 un tomo en folio y Pasta.

Tournefort, Joseph Pitton de

Contractus sub forma tabularum sistens Insitutiones rei herbaria juxta methodum modernarum

Frankfurt, 1715

96) Dalibard Flora Parisiensis Prodromus en Paris año de 1749 un tomo en $4^{\circ}$ y Pasta.

Dalibard, Thomas François

Florae Parisiensis prodromus

A Paris: chez Durand, 1749.

97) Le Jardinier Francois en Paris año de 1765 un tomo en octabo y Pasta.

Anónimo 


\section{LA BOTÁNICA EN EL JARDÍN DE MIGAS CALIENTES}

Le Jardinier François

Paris: Compagnie des Marchands Libraires, 1665.

98) Dilleni Catalogus Plantarum ex Pontecirca an conforti año de 1719 un tomo en $4^{\circ}$ y Pasta.

Dillenius, Joannes Jacobus

Catalogus plantarum...Cum appendice.

[S.I.]: impensis auctoris; prostat Francofurti ad Moenum: apud Joh. Maximilianum A Sande, 1719.

99) Bahuino des Plantes adibis sacntis Nomen habentibus.en Basilia año de 1591 un tomo en $4^{\circ}$ y Pasta.

Bauhin, Caspar

De plantis a divis sanctis've nomen habentis

Basilea: apud Conrad Uvaldkirch, 1591.

100) Histoire des Plantes del Europe en Leon año de 1753 en dos tomos en $4^{\circ}$ y Pasta.

Ville, Nicolas de

Histoire de plantes de l'Europe

A Lyon: chez Benoit Duplain, 1766. (¿Otra edición?) (1 ${ }^{\text {a }}$ ed. en Lyon, 1707)

101) Seguier Plantarum Beronenses en Verrone año de 1745 dos tomos en $4^{\circ}$ y Pasta.

Seguier, Jean François

Plantae Veronenses

Veronae: typis Seminarii, 1745, vols. I y II. (vol. III, 1754)

102) Pontederae Pisani tabularum Botanicarum en Patabi año de 1718 un tomo en Folio y Bitela. Pontedera, Giulio

Compendium tabularum botanicarum

Patavii: typis Seminarii: apud Joannem Manfrè, 1718.

103) Pontederae de Floris Naturalis en Patabi año de 1720 un tomo en $4^{\circ}$ y Bitela.

Pontedera, Giulio

Anthologia

Patavii: typis Seminarii: apud Joannem Manfrè, 1720.

104) Columnae Fitob.en Florencia año de 1744 un tomo en $4^{\circ}$ ymperial.

Colonna, Fabio

Phitobasanos

Florentaiae: I. P. Aere: \& typis Petri Caietani Viviani, 1744. (1 $1^{\text {a }}$ ed. en Nápoles, 1592)

105) Ambrosini de Plantis en biena año de 1666 un tomo en Folio y Bitela.

Ambrosini, Giacinto

Phytologiae: De plantis partis primae

Bononiae: Sumptibus Haeredum Evangelistae de Duciis, 1666. 


\section{SUSANA PINAR Y MIGUEL ANGEL PUIG-SAMPER}

106) Mateoli senensis Historia Plantarum et Medica en Benecia año de 1560 un tomo en folio tabla y filete negro.

Mattioli, Pietro Andrea Gregorio

Medici senensis Commentarii, in libros ses Pedacii Dioscoridis Anazarbei, de medica materia?

Venetiis, 1554.

107) Passaei Hortus floridus en el año de 1614 un tomo en Folio y Pergamino.

Passe, Crispijn de, Le Jeune

Hortus floridus

[Ultraiecti: ex officina Hermanni Borculoi et prostant apud Ioannem Iansonium bibliopolam Arnemiensis], 1614-1616.

108) Dorosteni herbarum Botanico en Francforte año de 1540 un tomo en folio y Bitela.

Dorstenius, Theodoricus

Botanicon

Francofurti: Christianus Egenolphus excudebat, 1540.

109) Schewzeri Itinera per elbetie en Ludoni año de 1723 dos tomos en $4^{\circ}$ y Pasta Ymperial que comprende 1.2.3.y $4^{\mathrm{a}}$ Parte.

Scheuchzer, Johann Jacob

Itinera per Helvetiae Alpinas regiones

Lugduni Batavorum: typis ac sumptibus Petri Van der Aa, 1723. $\left(1^{\mathrm{a}}\right.$ ed. del tomo $1^{\circ}$ en Londres, 1708)

110) Plukenete opera omnia Botanica en Londini año de 1720 y en otros años cuatro tomos en $4^{\circ}$ Ymperial y Pasta.

Plukenet, Leonard

Opera omnia botanica

Londres, 1720.

111) Hortus Sanitatis Medici en Labreje un tomo en folio y pergamino.

Anónimo

Ortus Sanitatis

Moguntia: Jacobus Meydenbach, 1491. (existe otra edición en Venecia, 1511)

112) Dodon. Historie des Plantes en Lacuelle en Ambers año de 1557 un tomo en folio y pergamino.

Dodoens, Rembert

Histoire des plantes

En Anvers: de l'imprimerie de Iean Loë, 1557.

113) Chabrai yncones Plantarum Estirpium en Colonia año de 1666 un tomo en folio y pasta.

Chabrey, Dominique

Stirpium icones et sciagraphia

Genevae: typis Phil. Gamoneti \& Iac. de La Pierre, 1666. 


\section{LA BOTÁNICA EN EL JARDÍN DE MIGAS CALIENTES}

114) Micheli Nova Plantarum Genera en Florencia año de 1729 un tomo en folio y Pasta Berde. Micheli, Pier Antonio

Nova plantarum genera

Florentiae: typis Bernardi Paperinii.., 1729.

115) Clusi Rariorum Plantarum Historia en antuerpia año de 1601 un tomo en folio y Bitela

L'Escluse, Charles de

Rariorum plantarum historia

Antverpiae: ex officina Plantiniana, apud Ioannem Moretum, 1601.

116) De Historia Estirpion en Basilie año de 1542 un tomo en folio ymperial y Bitela.

Fuchs, Leonhart

De historia stirpium

Basileae: in officina Isingriniana, 1542.

117) Dilleni Historia Plantarum et Moscorum en Hosoni año de 1741 un tomo en folio y pasta. Dillenius, Joannes Jacobus

Historia muscorum

Oxonii: e Theatro Sheldoniano, 1741.

118) Volkameri Flora Noribergensis Catalogus Plantarum en Norinberge año de 1700 un tomo en $4^{\circ}$ y Bitela.

Volckamer, Johann Georg, le fils

Flora Noribergensis

Noribergae: sumtibus Michaellianis: literis Knorzianis, 1700.

119) Fabi columnae Nincei en Romae año de 1616 un tomo en $4^{\circ}$ y pergamino.

Colonna, Fabio

Minus cognitarum rariorumque stirpium ekphrasis

Romae: apud Jacobum Mascardum, 1616.

120) Turre Historia Plantarum Driadum Amadriadum en Patabie año de 1685 untomo en folio y Bitela.

Torre, Giorgio dalla

Historia Plantarum

Patavii: Typis Petri Mariae, 1685.

121) Pomet Histoire General des Drogues en Paris año de 1694 un tomo en folio y Bitela.

Pomet, Pierre

Histoire générale des drogues

A Paris: chez Jean-Baptiste Loyson, \& Augustin Pillon, 1694.

122) Villa Ramillete de Plantas Medicinales en Burgos año de 1737 un tomo en $4^{\circ}$ y pergamino.

Villa, Fray Esteban de

Ramillete de plantas

En Burgos: Por P. Gomez de Baldivielsso, 1637. 


\section{SUSANA PINAR Y MIGUEL ANGEL PUIG-SAMPER}

123) Herman paradisus Batabus en Luduni año de 1705 un tomo en $4^{\circ}$ y Bitela.

Hermann, Paul

Paradisus Batabus

Lugduni Batavorum: apud Petrum van der Aa, 1705.

124) Teatrum Florae Ynquo año de 1633 en Paris un tomo en folio y Pasta.

Rabel, Daniel

Theatrum florae

Lutetiae Parisiorum: apud Petrum Firens, 1633. ( $1^{a}$ ed. en París, 1622)

125) Alpini de Plantes exoticis en Benecia año de 1656 un tomo en $4^{\circ}$ y pasta.

Alpino, Prospero

De plantis exoticis

(Venetiis: apud Guerilium), 1656. (1 $1^{\text {a }}$ ed. en Venecia, 1627)

126) Heist.Disertatio Inauguralis Medico Botanica en Tipis año de 1741 un tomo en $4^{\circ}$ y pasta. Sandhagen, Jodocus Edmundus

Dissertatio inauguralis medico-botanica de nominum plantarum mutatione utili ac noxia / quam praeside Laurentio Heistero..

Helmaestadii: litteris Schnorrianis, 1741.

127) Donati tratato de simplici, en Benecia año de 1631 un tomo en $4^{\circ}$ y pasta.

Donati, Antonio

Trattato de semplici

In Venetia: appresso Pietro Maria Bertano, 1631.

128) Indicis Botanica Plantarum en Bolonia año de 1753 un tomo en $4^{\circ}$ y pasta.

Monti, Gaetano Lorenzo

Indices botanici et materiae medicae quibus platarum genera hactenus instituta; simplicium quoque tam vulgarium quam exoticarum nomina, \& facultates summatin recensitur. Bononiae: ex typographia haelii a Vulpe, 1753.

129) Muntinguio Herba Bretanica en Asterdami año de 1681 un tomo en $4^{\circ}$ y pasta.

Munting, Abraham

De vera antiquorum herba Britannica

Amstelodami: apud Hieronymum Sweerts, 1681.

130) Lemeri traite unibersal de Es drogues en Paris año de 1714 un tomo en $4^{\circ}$ y Bitela.

Lemery, Nicolas

Traité universel des drogues simples

A Paris: chez Laurent d'Houry, 1714.

131) Fuchsi Historia estirpium en Basilea año de 1542 un tomo en folio y Bitela.

Fuchs, Leonhart

De historia stirpium

Basileae: in officina Isingriniana, 1542. 


\section{LA BOTÁNICA EN EL JARDÍN DE MIGAS CALIENTES}

132) Valentini Historia simplicium et India liter.en Franconforti año de 1716 un tomo en folio y Bitela.

Valentini, Michael Bernhard

Historia simplicium reformata

India literata

Francofurti ad Moenum: ex officina Zunneriana, apud Johannem Adamum Jungium, 1716.

133) Hortus floridus Gonense en el año de 1614 un tomo en folio y pergamino.

Passe, Crispijn de, Le Jeune

Hortus floridus

(Utrech: apud I. Iansonium), 1614-1616.

134) Teatri Botanici Gaspari Bauhini en Franforti año de 1620 un tomo en $4^{\circ}$ y Bitela.

Bauhin, Caspar

Prodomos. Theatri botanici

Francofurti: typis Pauli Iacobi, 1620.

135) Aincones Plantarum en Franconforti año de 1590 un tomo en $4^{\circ}$ y Bitela.

Theodorus, Jacobus

Eicones plantarum

Francofurti ad Moenum: [Nicolao Bassaeno], 1590.

136) Historia Plantarum Leonardo Thirneisero en Berlini año de 1578 un tomo en folio Ymperial y pergamino.

Thurneisser zum Thurn, Leonhard

Historia plantarum

Berlini: Excudebat Michael Henstzsche, 1578.

137). Joanis Georgi Kramer tentamen en Biena año de 1744 un tomo en folio y Pasta.

Kramer, Johann Georg Heinrich

Tentamen botanicum emendatum et auctum

Viennae Austriae: apud Leopoldum Joannem Kaliwoda, 1744. (1 ${ }^{\mathrm{a}}$ ed. en Dresde, 1728)

138) Roellio de Nature stirpium en Basilea año de 1537 un tomo en folio y pergamino.

Ruel (Ruelle?), Jean

De natura stirpium

Basileae: in officina Frobeniana, 1537. (1ª ed. en París, 1536)

139) Anselmi Boeti florum Herbarum en Brugis año de 1740 un tomo en $4^{\circ}$ y Bitela. Boot, Anselmus Boetius de

Florum, herbarum ac fructuum icones

Brugis Flandrorum: apud Ioannem Baptistam et Lucam Kerchovios, 1640.

140) Wepfero Historia et nosae en Basilea año de ...un tomo en $4^{\circ}$ en encerado Berde. Wepfer, Johann Jakob 


\section{SUSANA PINAR Y MIGUEL ANGEL PUIG-SAMPER}

Cicutae aquaticae historia

Basileae: apud Joh.Rodolphum König, 1679.

141) Herbario Nobo Dicastore Durante en Benecia año de 1617 un tomo en folio y pergamino. Durante, Castore

Herbario novo

In Venetia: appresso li Sessa, 1617. ( $1^{\mathrm{a}}$ ed. en Roma, 1585)

142) Batabo-orto Academico en Ludoni año de 1727 un tomo en $4^{\circ}$ y Bitela.

Boerhaave, Herman

Index alter plantarum, quae in Horto academico...

Lugduni Batavorum: apud Janssonios Vander Aa, 1727.

143) Monti de Planti Bari Indici en Bolonia año de 1724 un tomo en $4^{\circ}$ y Bitela.

Monti, Giuseppe

Exoticarum simplicium medicamentorum varii indicis.

Bononiae, 1724.

144) Bocone Museo de Plante Rare en Benecia año de 1697 un tomo en $4^{\circ}$ y Bitela.

Boccone, Paolo Sylvio

Museo di piante rare

In Venetia: per Io. Baptista Zuccato, 1697.

145) Konig Renum Begetabilea en Bas..año de 1708 un tomo en $4^{\circ}$ y Bitela.

Koenig, Emanuel

Regnum vegetabile

Basileae: typis \& impensis Emanuelis König, senioris, 1708.

146) Linei Hortus cliforcianus en Asterdan año de 1737 un tomo en folio y Bitela.

Linné, Carl von

Hortus Clifforticanus

Amstelaedami: [s.n.], 1737.

147) Ludwig Reyne Begetabilum en Lisia año de 1742 un tomo en $4^{\circ}$ y pasta.

Ludwig, Christian Gottlieb

148) Prosperi Alpini de Plantis aegictis en Benecia año de 1592 un tomo en $4^{\circ}$ y pergamino.

Alpino, Prospero

De plantis Aegypti

Venetiis: apud Franciscum de Franciscis Senensem, 1592.

149) Cornuti Historia Plantarum en Paris año de 1635 un tomo en $4^{\circ}$ y Bitela.

Cornut, Jacques Philippe

Canadensium plantarum historia

Parisiis: venundantur apud Simonem Le Moyne, 1635. 


\section{LA BOTÁNICA EN EL JARDÍN DE MIGAS CALIENTES}

150) Batabi Horti Academico en Ludoni año de 1687 un tomo en $4^{\circ}$ y Bitela.

Hermann, Paul

Horti academici Lugduno-Batavi catalogus

Lugduni Batavorum: apud Cornelium Boutesteyn, 1687.

151) Bocone Yncones Descripciones Rariorum Plantarum en Londini año de 1674 un tomo en $4^{\circ}$ y pasta.

Boccone, Paolo Sylvio

Icones et descriptiones rariorum plantarum

[Oxford]: e Theatro Sheldoniano: prostant apud Robertum Scott bibliopolam Londinensem, 1674.

152) Historia estirpium de Plantas en Luduni año de 1551 un tomo en $4^{\circ}$ y pergamino.

Fuchs, Leonhart

De historia stirpium comentarii insignes

Lugduni: apud Balthazarem Arnolletum, 1551. (1 ${ }^{a}$ ed., 1542)

153) Magnol Botanico Monspeliense en Luduni año de 1676 un tomo en $4^{\circ}$ y pasta.

Magnol, Pierre

Botanicum Monspeliense

Lugduni: ex officina Francisci Carteron, 1676.

154) Haller Flora Henensis en Bienae año de 1745 un tomo en $4^{\circ}$ y pasta.

Ruppius, Heinrich Bernhard

Flora jenensis. Henrici Bernhardii Ruppi ex poshumis auctoris schedis et propriis observationibus aucta et emendata accesserunt plantarum rariorum novae icones.

Jenae: Sumptibus Christ. Henr. Cunonis, 1745. Ed. de Albert Haller.

155) Magnoli Botanico Monspeliense en Luduni año de 1676 un tomo en $4^{\circ}$ y pasta. (Repetición de 153)

156) Hermani escola Botanica sibe Catalogus Plantarum en Asterdami año de 1689 un tomo en $8^{\circ}$ y pasta.

Tournefort, J.P.y Hermann, P.

Schola botanica,..., Paradisi Batavi prodromus...

Amstelaedami: apud Henricum Wetstenium, 1689.

157) Teatri Botanici Gaspar Bahuino en Basilea año de 1623 un tomo en $4^{\circ}$ y Bitela.

Bauhin, Caspar

Pinax theatri botanici

Basileae Helvet.: sumptibus \& typis Ludovici Regis, 1623.

158) Gouan Hortus Regius en Luduni año de 1762 un tomo en $4^{\circ}$ y pasta.

Gouan, Antoine

Hortus Regius Monspeliensis

Lugduni, 1762. 


\section{SUSANA PINAR Y MIGUEL ANGEL PUIG-SAMPER}

159) Huerto malaberico impresos en Asterdan año de 1778 en seis tomos y otro tomo de su indice en pasta.

Rheede Tot Draakenstein, Hendrik Adriaan van

Hortus Malabaricus

Amstelodami: sumptibus Joannis van Someren, 1678-1703.

160) Gerardo Runphio Herbario Anboinense en Asterdan año de 1743 en seis tomos en pasta de A folio.

Rumpf, Georg Eberhard

Herbarium Amboinense

Amstelaedami: apud Meinardum Uytwerf, 1750 (1741-50). 6 vol.

161) Santiago Serad, Dillenius, Hortis el thamensis en Londres año de 1732 dos tomos de a Folio en Pasta.

Dillenius, Joannes Jacobus

Hortus Elthamensis

Londini: sumptibus auctoris, 1732 .

162) Basilio Beslero Huerto Eistense en Basilea año de 1612 y otros años en tres tomos de a folio ynperial.

Besler, Basil

Hortus Eystettensis

[Nuremberg]: [s.n.], 1613.

163) Jacobi Brein ex soticarum Plantarum año de 1677 un tomo en folio y pasta.

Breyn, Jakob

Exoticarum plantarum centuria prima

Gedani: typis, sumptibus \& in aedibus autoris, 1678.

164) Baillant Botanico Parisiense en Leiden año de 1727 un tomo en folio y pasta. Vaillant, Sébastien

Botanicon Parisiense

A Leide; et à Amsterdam: chez Jean et Herman Verbeek: et [chez] Balthazar Lakeman, 1727.

165) Gesneri opera Botanica en Norinberge año de 1753 un tomo en folio ynperial y pasta. Gesner, Conrad Opera botanica (pars prima)

Norimbergae: impensis Jo. Mich. Seligman...: typis Jo. Josephi Fleischmanni, 1753.

166) Plumier Descripciones de Plantas en Paris año de 1693 un tomo en folio y Bitela. Plumier, Charles

Description des plantes de l'Amerique

A Paris: de l'Imprimerié royale, 1693. 